\title{
SOBRE ASUMIRSE VULNERABLE, SER VULNERABLE Y LA VIVENCIA DE LA VULNERABILIDAD.
}

\section{Comprensión de la vulnerabilidad desde el enfoque fenomenológico-existencial}

\author{
Olga Leonor Hernández Bustamante ${ }^{1}$ \\ Johana Carolina De La Ossa Sierra ${ }^{2}$ \\ Amelia del Cristo Eljadue Rizcala
}

\section{Resumen}

Hacer frente a la propia existencia implica, en el intento de preservarnos, aceptar la necesidad de poder y autoafirmación que emerge con un acompañante: la certeza de la impotencia de no poder ser o no poder afirmarse siempre en todos los escenarios. La angustia que esta dualidad presenta, puede interrumpir la propia actualización o, por el contrario, encaminar la voluntad hacia la afirmación o preservación de los propios valores. Desde la comprensión del enfoque fenomenológico-existencial, el lector de este capítulo encontrará una revisión teórica de lo que significa para

$1 \quad$ Doctoranda en Psicología Universidad de Flores (Argentina)- MG en Psicología y Especialista en Psicología Clínica Enfoque Fenomenológico-Existencial (Universidad del Norte) Especialista en Derechos Humanos (ESAP). Especialista en Docencia (CECAR). Psicóloga (Universidad de San Buenaventura - Medellín). Docente investigadora en Corporación Universitaria del Caribe - CECAR - Sincelejo. Grupo de investigación Dimensiones Humanas - Líneas Desarrollo cognitivo, salud mental y neuropsicología y Vulnerabilidad social y grupos poblacionales.e-mail olga.hernandezb@cecar.edu.co

2 Magister psicología y Especialista en Psicología Clínica Enfoque Fenomenológico - Existencial (Universidad del Norte). Psicóloga (Universidad del Norte). Docente tiempo completo e Investigadora Línea Clínica del Grupo de Investigación en Psicología (GIPSI) Universidad de San Buenaventura - Cartagena. e-mail: jdelaossa@usbctg.edu.co

3 Magister psicología Enfoque Fenomenológico - Existencial (Universidad del Norte). Psicóloga (Universidad del Norte). Docente e Investigadora Línea Clínica del Grupo de Investigación en Psicología (GIPSI) Universidad de San Buenaventura - Cartagena. e-mail:amelierizcala27@gmail.com 
una persona la vulnerabilidad desde dos lugares: asumirse vulnerable se presenta como la vulnerabilidad de adentro-hacia-afuera, es decir, tomar una postura de donde la persona se protege, ancla, o decide frenar su proceso de actualización; y ser vulnerable se presenta como la vulnerabilidad de afuera-hacia-adentro, donde la persona se enfrenta a un contexto o situación amenazante que lo llevan a la disminución de las posibilidades hasta ese momento conocidas. Esperamos que la lectura de este capítulo sea clarificadora y plantee comprensiones, y, al mismo tiempo, interrogantes que orienten discusiones constructivas para la comprensión del contexto y del modo de ser de las personas desde y ante este.

Palabras clave: vulnerabilidad, angustia, tendencia actualizante, ansiedad, incongruencia, frustración, impotencia, valoración, postura.

\section{Abstract}

To face one's own existence implies, in the attempt to preserve us, to accept the need for power and self-affirmation that emerges with a companion. The certainty of the impotence of not being able to be, or not being able to always affirm in all scenarios. The anguish that this duality presents can interrupt the actualization itself or, on the contrary, direct the will towards the affirmation or preservation of one's own values. From the understanding of the phenomenologicalexistential approach, the reader of this chapter will find a theoretical review of what it means for a person to be vulnerable from two places: to assume vulnerable is presented as the vulnerability of inside-out, that is, to take a stance from where the person protects, anchors, or decides to stop their updating process; and being vulnerable presents itself as the vulnerability of the outside-towards-inside, where the person faces a threatening context or situation that leads to the diminution of the possibilities known until that moment. We hope that the reading of this chapter will clarify and raise understandings, and, at the same time, questions that guide constructive discussions to understand the context and the way of being of people from and before it.

Keywords: vulnerability, anguish, updating trend, anxiety, incongruity, frustration, impotence, assessment, posture. 


\section{A modo de introducción}

\section{Soy humano, y nada humano me es ajeno}

Terencio

La vulnerabilidad, en su acepción más amplia, es comprendida como un estado de susceptibilidad al daño (Adger, 2006), que incorpora las tensiones a las que está expuesto un sistema, su sensibilidad y su capacidad de adaptación. Esto implicaría que la vulnerabilidad en la experiencia de una persona expone y amenaza la capacidad de esta para hacer frente a una situación que interpreta como potencialmente amenazante, y, por lo tanto, le impide adaptarse y transformarse.

Al respecto, Romero (2003) se pregunta entonces “¿Cuáles son los factores personales que llevan a las diversas formas de desencuentro humano?" (p. 110). La pregunta se dirige al desencuentro como modo de ser vulnerable al estar en asintonía, en ruptura, con las posibilidades de realización personal en los diversos planos: corporal, sexual, relaciones interpersonales y afectivas, trabajo, subjetividad y metafísico. Sucede, en estas situaciones, un interjuego entre factores materiales (socio-económicos, ambientales) y la configuración psicológica del individuo (los recursos que su estructura básica le ofrece). Los factores materiales influyen en la configuración de la personalidad, pero también la personalidad media en el modo de percibir, evaluar y tomar posición en el contexto. Plantea el autor que vivencias negativas causadas por situaciones críticas, conflictivas, estresantes o deprimentes devienen en periodos de crisis marcados por un desequilibrio en la esfera afectiva, así como también sucede la crisis como resultado de características negativas de la personalidad, como la impulsividad, la pasividad, la falta de persistencia, la ausencia de propósitos, rigidez e indolencia.

Por tanto, al enfrentarnos al concepto de asumirse vulnerable o ser vulnerable en un contexto dado, implica de forma obligada el surgimiento de dos interrogantes: 1. ¿De qué modo se comprende a sí misma una persona para relacionarse con una experiencia desde la noción de sentirse vulnerable y, por tanto, disminuida en sus posibilidades de afrontarla? 2. ¿De qué manera un contexto puede disminuir a una persona hasta hacerla 
dudar de sus propias capacidades y potencial? La primera pregunta implica una vulnerabilidad que llamaremos de adentro-hacia-afuera (asumirse vulnerable) y la segunda pregunta una vulnerabilidad que llamaremos de afuera-hacia-adentro (ser vulnerable en un contexto dado).

Los guiones entre las palabras implican la comprensión de un ser humano en interacción permanente con su contexto, con lo que es y potencialmente puede ser, en un lugar y tiempo, además con lo que su contexto le permite ser y desarrollar en potencia. En relación con esto, (de Castro, 2010) nos pide tener presente que toda experiencia y creación del ser humano está ubicada, enraizada, basada y contextualizada en la total relación, orientación y postura de la persona en el mundo y en el significado que esta pretenda reafirmar o preservar para sentir que tiene algún valor como persona. También, (Martínez, 2010) recuerda al retomar el concepto de Dasein (ser-en-el-mundo), que la realidad humana debe encontrarse en el momento preciso del encuentro del ser y el mundo. No hay humanidad que anteceda este encuentro: la existencia humana es una relación, es decir, un ser que se encuentra en un mundo en el que hay otros como él que se le asemejan, a la vez que se diferencian de él. De esta manera, el foco se pone en la manera en que la persona en relación esta junto con los otros co-creando la experiencia intersubjetiva del aquí-y-ahora relacional en el que se encuentran.

Una mejor visión de este elemento relacional se obtiene al comprender al ser humano como un ser-en-el-mundo. Es en el mundo donde se encuentra con el otro, con lo otro lo que otorga un sentido en cuanto coexistencia y desde donde se construye y deconstruye permanentemente el mundo singular de cada persona. Desde allí, surgen 3 regiones del ser (Rispo, 2001): SER - correspondiente al Umwelt o mundo circundante (el cuerpo, lo factico y los acontecimientos, los accidentes, el hábito, lo familiar, las circunstancias o la historia); EN EL - relacionado al Mitwelt o el mundo con el otro; MUNDO - comprendido como el Eigenwelt o mundo propio, donde se manifiesta la singularidad del ser humano. 


\section{Vulnerabilidad de adentro-hacia-afuera}

Desde el enfoque humanista-existencial la idea de la actualización y autorrealización son conceptos centrales que evidencian la ruta de un organismo total y consciente hacia su propio desarrollo. Se entiende, entonces, que este enfoque trabaje desde la premisa de la confianza en lo que el ser humano ES en su totalidad y por tanto en la orientación que puede otorgar a su existencia si es congruente. Es decir, si la persona es capaz de conocerse profundamente, conoce por tanto aquello que desea y puede dirigir responsablemente su deseo a voluntad.

Respecto de esta tendencia actualizante, Horney aborda la idea de la autorrealización afirmando que "En el hombre son inherentes fuerzas constructivas deevolución queleimpulsan a desarrollarsuspotencialidades... es decir que el hombre por su misma naturaleza y propio acuerdo lucha por realizarse" (1991, p. 11). A esta fuerza potencial al desarrollo se le impone que la persona sea sincera consigo misma, es decir, que tenga un conocimiento cada vez mayor de sí como medio de liberar la fuerza del desarrollo espontáneo y, por lo tanto, asuma la plena responsabilidad de sí mismo. Para esta autora, el neurótico se entrega a la "oscura egolatría" y termina atribuyendo a los demás todos sus inconvenientes.

La vulnerabilidad, entonces, se asume como una interrupción de lo que Rogers (2014) llama tendencia actualizante, comprendida como la tendencia innata de un organismo a desarrollar sus potencialidades, siendo el organismo en su totalidad - y no una porción de este- quien muestra esta tendencia. Para Rogers (2014, p.25), cuando hay acuerdo entre el yo y el organismo en su totalidad, la tendencia actualizante opera de modo unificado. En un organismo para el cual la tendencia a la actualización es garantía de supervivencia, la posibilidad de ser dañado y ver interrumpida su posibilidad de avance y crecimiento se vivencia de forma extremadamente angustiante. Quien se actualiza, obtiene desde su experiencia herramientas que le permiten enfrentar, en un bucle permanente de aprendizaje, situaciones que requieren hacer uso de las comprensiones obtenidas en el recorrido previo, pero siempre en una orientación hacia adelante.

De este modo, la incongruencia entre el yo y la experiencia (es decir entre lo que la persona quiere ser o cree que es y lo que realmente es) lleva a la incomprensión de sí mismo y, por consiguiente, a la interrupción 
del proceso de actualización (quien no sabe quién/que es, no sabe qué camino tomar). En esta situación, sometida queda la persona a la confusión y tensión internas y a la aparición de conductas discordantes. Esta visión rogeriana lleva a la comprensión de la vulnerabilidad como el estado de incongruencia entre el yo y la experiencia, estando el ser humano en este estado sin tener conciencia de ello.

Es así como la persona está potencialmente vulnerable a la amenaza, la angustia y desorganización que la incongruencia misma conlleva, pues —ante esta situación no comprendida, no percibida y no llevada a la conciencia- el yo corre el riesgo de desorganización al caer en la contradicción. Así, afirma Rogers "si una nueva experiencia pone en evidencia la discrepancia con tal claridad que el individuo no puede dejar de percibirla conscientemente, se sentirá amenazado y su concepto del yo quedará desorganizado por esa experiencia contradictoria e inadmisible" (2014. p.35). Es necesario clarificar algo: la angustia no es en sí misma un problema a eliminar, la dificultad surge ante la contradicción, la incongruencia y la interrupción de la tendencia actualizante. La vivencia de la angustia es ineludible en el ser humano y es reflejo de las limitaciones y el reconocimiento de nuestra condición humana y mortal. Riemann (1996) explica que la angustia es vivenciada de manera propia y particular por cada ser humano concreto, es decir, que depende en alguna medida de circunstancias individuales y ambientales.

La ansiedad ilumina aquellas experiencias que vivimos y que, de otro modo, las pasaríamos por alto (May, 1989). En ese sentido, la vivencia de ansiedad no es exclusivamente negativa, sino, que en palabras de May (1963), presupone una antena necesaria para captar la batalla psicológica que está por librarse. En situaciones de vulnerabilidad, la ansiedad emerge como respuesta a la experiencia inminente del no-ser (muerte psicológica y física) (May, 1977), y puede resultar una experiencia abrumadora para el ser humano, dado que nubla la percepción, generando la sensación de estar atrapado por la situación de amenaza (May, 1989), lo que le impide percatarse de sus potencialidades y diversas posibilidades de ser.

Está vivencia de la angustia, si bien es particular a cada ser humano, nos enfrenta a la antinomia de movernos entre exigencias fundamentales que se vivencian como extremas. Riemann (1996, p.16) explica que la primera exigencia es la de la individuación, donde la persona ha de afirmar 
y constituirse en un individuo único y delimitado. La segunda exigencia es la de integrarnos a un conjunto superior, abriéndonos y tendiendo lazos con el no-yo que esta fuera de nosotros. Estas dos exigencias que se vivencian como opuestas constituyen la primera antinomia que es la de la individuación versus la integración. La tercera exigencia, es la de la constancia y duración, es decir, proyectarnos para el futuro, estableciéndonos y buscando el logro de objetivos. La cuarta exigencia es la del cambio y la transformación, es decir, el estar dispuestos a modificaciones y renuncias. Estas dos exigencias constituyen una nueva antinomia: la de la permanencia versus la mutación.

A partir de allí, Riemann (1996, p. 19) define cuatro formas básicas de la angustia: 1. La angustia ante la renuncia de uno mismo, vivida como la dependencia y pérdida del yo; 2. La angustia de llegar a ser uno mismo, lo cual equivale al aislamiento; 3. La angustia a la transformación por la transitoriedad vivida como inseguridad; y 4. La angustia ante la necesidad de fijarse vivenciada como falta de libertad. El predominio de cualquiera de las formas básicas, da lugar a modos de estar en el mundo o estructuras de personalidad, mismas que tienen manifestaciones sanas y adaptativas hasta trastornos. Enmarcado en estos modos básicos, la persona moldea lo que ES al asumir una postura, comportamiento y actitud ante la vida.

De modo que la angustia, para cada ser humano concreto, supone una doble cara. Puede activar o paralizar. La angustia activa al ser humano cuando al aceptarla se avanza en los modos de superarla y afrontarla recorriendo así el camino a la maduración. Por el contrario, la angustia es paralizante y deviene en vulnerabilidad cuando se opta por rehuirla, evitando enfrentar y afrontar aquello de lo cual deriva. En este caso, se está frente a un ser humano que estanca su proceso de desarrollo y bloquea el camino a su maduración. La angustia, entonces, puede perder su cualidad activadora y manifestarse de modo tal que la persona es incapaz de soportar su propia ansiedad. Anota Riemann (1996, p20) que, en situaciones excepcionales, se puede, incluso, "romper el índice de tolerancia", lo que lleva a la persona a reaccionar con pánico. Entonces, toda vivencia que "conflictúe, bloquee, desgaste, tensione y se imponga" a un individuo, limitando su libertad, provoca efectos en su modo de ser en el mundo, inhibiendo su potencial de desarrollo y realización.

Son diversas las situaciones que conflictuan al ser humano y que generan en él sensaciones de apatía, insatisfacción y vulnerabilidad, pues 
presuponen una amenaza directa a su identidad y, con esto, a sus sentidos de ser (May, 1988, 1990). Entre ellas encontramos la pérdida y el fracaso que suele generar sentimientos de tristeza, desánimo y depresión, haciendo que el ser humano sienta que su vida pierde luminosidad y sentido llevándolo a desligarse de aquello que ayer consideró significativo. Otra de ellas es la ansiedad que sobreviene al confrontarnos con la posibilidad del estancamiento en el propio desarrollo personal, al sentir que se es incapaz de hacerle frente a las exigencias de la vida, lo cual suele desorientar al ser humano y generar sensaciones de minusvalía y desesperación. Cada uno de estos son sentimientos negativos que generan estados de malogro y una profunda ansiedad, la cual, si se enfrentan constructivamente, puede ser la puerta al desarrollo personal. Bien afirma May (1988) que el fin de la vida humana no es acabar con los errores, sino levantarse cada día para encontrarse con los desafíos que comporta la existencia en sí misma, y, con ella, asumir nuestro destino a través del empleo de la libertad para hacerle frente al reto mismo de estar vivo.

Lo negativo y lo positivo coexisten de manera desigual en todas las personas (Romero, 2003). Sin embargo, toda vivencia que genere conflicto, bloquee, desgaste, tensione y se imponga al sujeto, restringiendo su sentimiento de libertad, provoca efectos en sus actividades, inhibiendo su potencial de desarrollo y realización. Lo decisivo, para que la situación no entre por la sendas del malogro, es el sentimiento de autoconfianza que posee, lo cual acredita que aún se tienen posibilidades en medio de la carencia y el conflicto (Romero, 2003).

Ahora bien, la forma como se integra el trauma a la totalidad de la experiencia depende, en gran medida, de la valoración que la persona hace de su situación de vida (de Castro Correa \& García Chacón, 2011), dado que, por un lado, podría encontrar en ella la posibilidad de continuar su desarrollo personal o, por el contrario, sumirse en el estancamiento (Riemann, 1996), en la medida en que su proyecto de vida fracasa o se ve malogrado (Romero, 2003), generando sensaciones de frustración e insignificancia (Cyrulnik, 2009). Dicha sensación surge, precisamente, de experimentar que los propios recursos psicológicos no son suficientes para enfrentar la batalla psicológica que se experimenta y el inminente dolor que comporta (May, 1963), al ver tambalear el proyecto de vida que se ha venido forjando. Lo que la persona haga con esa sensación de vulnerabilidad, 
dependerá de la orientación de su intencionalidad. Puede compensarla expansivamente siendo dominante y competitivo, o puede renunciar a su libertad y buscar una dependencia morbosa a través de una intencionalidad hiperconstrictiva.

El enfrentamiento de nuestro destino implica reconocer nuestra condición de vulnerabilidad y, con ello, la sensación de desesperación que sobreviene al ver frustrado nuestro proyecto de vida o amenazada nuestra estructura existencial. Sin embargo, confrontarla implica percatarse de nuestra fuerza interior, reconciliarnos con nuestro destino y, con ello, ser capaces de enfrentar nuestra realidad, asumir la riendas de nuestra vida, dándole vuelta al conformismo, haciendo frente a nuestra condición libre y responsable (May, 1989) dejando de lado lo conocido, aquello que nos genera cierto grado de seguridad, aunque sea reducido para aventurarnos y enfrentar nuestro propio potencial creador (May, 2000).

\section{Vulnerabilidad de afuera-hacia-adentro}

Romero (2003, p. 155) brinda una posición clarificadora: si bien hay aspectos afectivo-emocionales que perturban los propósitos de las personas, no es suficiente estar sufriendo sentimientos negativos para ser neurótico. Se debe tener presente que las reacciones surgen ante factores externos, que alteran el modo habitual que la persona tiene de regular su interacción con el mundo. Se cuenta allí la culpa por faltar a deberes éticos; por haber descuidado el propio desarrollo; la tristeza y el desánimo asociados a pérdidas y fracasos Romero (2003, p.116); y la ansiedad ante las exigencias que ponen a la persona en situación de impotencia, vivida de forma inquietante y tensionante.

La vivencia de la impotencia nos lleva a lo planteado por May (1974, p.22), cuando afirma que el poder (palabra que deriva de posse: ser capaz) es esencial para toda cosa viviente. Esto, debido a que el ser humano debe garantizar su supervivencia y, para ello, enfrenta permanentemente fuerzas que se le oponen. La capacidad del niño, entonces, de hacer frente a las necesidades se convierte en la lucha por la autoestimación y el sentido de significación en cuanto persona, complementa May, afirmando que "el avanzar hacia el autorreconocimiento implica el poder decir yo soy" (p. 
22). Es así, como en la existencia humana entran en conflicto el poder y la impotencia; el poder es asumido bajo una connotación negativa (de maldad y dominación) y, al tiempo, la realidad de fuerzas opuestas que me evidencian la impotencia es una realidad dolorosa y difícil de enfrentar. Para May, no enfrentar la necesidad de poder que se hace evidente en toda existencia humana se explica porque hacerlo visible expone su contraparte: la impotencia; es decir, la propia vulnerabilidad.

La persona se siente vulnerable cuando pierde la ruta de orientación de su propio deseo; cuando, debido a elementos que escapan a su control, su proyecto se ve frustrado. "Cuando a un individuo se lo incapacita para defenderse por sí mismo social o psíquicamente, como en la esclavitud, ¿dónde va a parar su poder? Nadie puede aceptar la impotencia completa a menos que este muerto" (May, 1974, p.108). Aquel cuyo ambiente y sus exigencias lo vulneran, ve disminuido su potencial de desarrollo y las posibilidades que tiene a la mano para hacer frente a su propia existencia. Un ambiente que me disminuye es aquel que amenaza aquello que para mí es un valor central y, por tanto, la tendencia natural estará en proteger aquello que valoro, aun a costa de mi propio desarrollo.

Es así como la razón psicológica de la vida de un individuo es lograr autoestimación y sentido de significación como persona: ser capaz de decir yo soy, de afirmarse en su mundo interior, mediante la capacidad de hacerse valer y de hacer frente a las necesidades, creando, así, significado. Sin embargo, una característica en la sociedad actual es que el hombre tiene muy poca confianza auténtica en su poder de significar algo; así, se va despersonalizando y perdiendo el sentido de responsabilidad personal. Como consecuencia de esta impotencia, surge la violencia: "la violencia echa raíces en la impotencia y la apatía" (May, 1974, p.25).

La forma en que una persona asume y se adapta a las tensiones a la que está expuesto, depende de su postura intencional ante dicha situación. Es decir, la vivencia y respuesta a la vulnerabilidad es una postura que depende de la orientación de la intencionalidad. De esta manera, aquellas situaciones donde la persona se asume vulnerable, implican una postura de debilidad asumida frente a los demás, por la capacidad de herirnos que nosotros mismos le otorgamos y la consecuente sensación de indefensión de la persona. Por tanto, surgiría la impotencia de la persona de poder autoafirmarse y autodeterminarse. 
En este orden de ideas, no importa cuán grande o cuántos sean los determinantes que se imponen frente a las situaciones traumáticas. La decisión de cómo enfrentarlos, dependerá siempre de la valoración consciente de dichos determinantes captando su significado. A partir de ahí, nuevas posibilidades empezaran a emerger, lo cual posibilita la realización constructiva de proyectos; dado que el acto de valorar conscientemente es una expresión de libertad, que no elimina determinismos del pasado, biológicos y ambientales, es decir, que valorar no ayudará a erradicar las situaciones traumáticas, pero permite que la persona comprenda el papel de dichos determinismos en su vida actual. De ahí, la importancia de ofrecer una relación terapéutica que facilite al paciente aprender a entrar en contacto y relacionarse conscientemente consigo mismo, como ser-en-elmundo (de Castro Correa \& García Chacón, 2011).

Ahora bien, la agresión es más que la simple intención de agredir o someter al otro: presupone la necesidad de enfrentar el conflicto manifiesto propios de toda vivencia de amenaza; implica un arrojarse contra aquello que se presenta como adversario. Su meta es causar un cambio en el poder, en los intereses propios, las necesidades afectivas y en aquello que consideramos valioso para nuestra vida. En este sentido, la agresión puede tomar dos caminos. En una primera instancia, puede ser constructiva, orientándose a la afirmación del propio potencial, pero sin la intención de dañarse o dañar al otro; y, en una segunda instancia, puede ser neurótica, dirigiéndose a someter al otro bajo imposición, por lo que suele estar motivada por sensación de angustia, culpa y frustración, en cohesión con la necesidad de herir y generar sufrimiento, con la intención de protegerse uno mismo de aquella situación que presupone una amenaza para las posibilidades de ser y generar la sensación de valía que el poder le otorga al ser humano (May, 1974).

Sin embargo, ¿qué sentido tienen la aparición de la agresión neurótica? Según Fromm (2006), este tipo de violencia, cuyo eje central es someter al otro, ya sea a través de la sumisión o la venganza, genera una ganancia afectiva para el ser humano, pues, en términos de intencionalidad, la afirmación vengativa del sí mismo le permite al ser humano no enfrentarse directamente a la amenaza de hundirse y, con ello, perder su sentido del yo y por ende su identidad. En palabras de May (1974), su sentido de valer como persona. 


\section{El rol del terapeuta}

El enfrentamiento de la crisis implica — tanto para el terapeuta como para el paciente- enfrentar su situación de fragilidad ante las tragedias de la existencia, pues el rol de sanador del terapeuta no lo exonera de vivir en carne propia el sufrimiento. En este sentido, solo cuando el terapeuta está dispuesto a confrontar su propia situación existencial, puede acompañar al paciente a confrontar la propia. Esto no implica la resolución completa del conflicto psicológico, sino el reconocimiento de las condiciones inexorables de ser humano. En palabras de Yalom (2002): un compañero de viaje de su paciente.

El terapeuta deberá ser lo suficientemente empático y acogedor para poder acompañarlo hasta que pueda asumir una postura, de manera que, avanzando al ritmo del paciente, le ofrezca espacios para que pueda desarrollar los recursos psicológicos necesarios para enfrentar la crisis que implica toda vivencia traumática (Cyrulnik, 2009). Y, en ese sentido, pueda ir ganando autonomía y, con ello, relacionarse conscientemente con su situación de aislamiento existencial, integrando progresivamente la experiencia traumática a su existencia presente (Romero, 2003), ya sea la pérdida de alguien o algo significativo, la vivencia de un trauma emocional o, incluso, la sola sensación de sentir que la existencia en sí misma no es lo suficientemente significativa (Nietzsche cita por May, 1990). En el espacio terapéutico, es necesario, además, habilitar a la persona para admitir sus propios sentimientos individuales de impotencia y enojo, de tal forma que recupere para sí valores esenciales para nuestra condición humana como la autoafirmación y la autoaceptación.

Se trata, entonces, de que el encuentro terapéutico se convierta en elementos catalizador para los cambios, ofreciéndole al paciente un espacio en donde puede reconocer su situación de malogro existencial y, en este sentido, tomar conciencia de lo que le ocurre eventualmente, respetando el ritmo del paciente sin forzarlo. No se trata de que el terapeuta asuma una postura pasiva o permisiva en relación con el proceso terapéutico del paciente, sino, más bien, de ser capaz de lidiar con la propia ansiedad y respetar el ritmo que el paciente lleva en lo que respecta al enfrentamiento de su conflicto existencial, acogiendo el sentido genuino del dolor, desde la posibilidad de ofrecerle al paciente una relación basada en la aceptación y el apoyo, sobre todo de una persona que lo conoce tan íntimamente, lo 
cual es enormemente alentador (Yalom, 2002; Yalom \& Elkin, 2007). Es necesario tener en cuenta que retomar su deseo y su proyecto le implica reconocer su limitación, asumir y reformular la propia existencia.

En este sentido se propone que el terapeuta logre empatizar con el dolor del otro y sus experiencias traumáticas, con fin de que no solo pueda captar el sentido de la misma, sino ofrecerles uno en el que se lo convide a ser él mismo, siendo acogido, aceptado, comprendido y orientado, a fin de que pueda, en el marco del encuentro terapéutico, encontrar un espacio en que explora sus tensiones y dilemas existenciales, al lado de un compañero de viaje (Yalom, 2009) que lo insta constantemente a la autoexploración de sus potencialidades y nuevas posibilidades de ser. En palabras de Rogers (1981), ofrecerle un espacio en donde explore su vida, sus experiencias traumáticas, para que, una vez las confronte y reconozca como propias, pueda aventurarse en la construcción de nuevas metas, nuevos significados. Y logre resignificar su proyecto de vida, al asumir la propia responsabilidad en el enfrentamiento del hecho traumático como un determinismo que, si bien no eligió vivir, si es su elección: la forma en como lo integra a la totalidad de su experiencia de allí en adelante (Yalom, 2011).

Así como en la calidez emocional de la relación terapéutica empieza a experimentar un sentimiento de seguridad, a medida que encuentra que, cualquiera sea la actitud que exprese, se lo comprende casi de la misma manera en cómo la percibe y significa, esto le permite al paciente mostrase en la relación terapéutica tal cual es (Rogers, 1981; Yalom, 1997), logrando, en este sentido, resonar afectivamente en la vivencia del paciente (Romero, 2003), lo que le permite penetrar poco a poco en la experiencia de dolor que este vivencia y eventualmente captar su sentido (May, 2000).

Según Yalom (2009), el éxito terapéutico se asocia a ser genuino, tener empatía y mantener una actitud positiva e incondicional hacia el paciente, quien, en última instancia, está sufriendo por su situación de malogro existencial, lo cual implica crear un vínculo basado en la conexión, fijándonos ante todo en la experiencia del ser humano que tenemos al frente, más que en los roles que cada uno asume en la diada terapéutica.

Se trata, entonces, de ofrecerle al paciente una relación terapéutica que supera las limitaciones de la técnica para otorgar presencia, la cual se traduce en acoger al otro como un "tú" y esto implica establecer un vínculo 
cuya calidez afectiva está basada en lo que Villegas (2013) llama "Amor Terapéutico". Dicho movimiento afectivo implica permear la relación de autenticidad y realidad, dejando de lado las barreras artificiales que los roles suelen otorgar a la diada terapéutica. Actuando de buena fe, basando la cura en el contacto, y no solo en el conocimiento técnico con el que se pueda contar (Yalom, 2011). Considerando al otro no como un objeto para analizar, sino como una persona a la que hay que comprender, desde sus sensaciones de insignificancia, vulnerabilidad, apatía, dolor y minusvalía, ofreciéndole una conversación de existencia a existencia, de persona a persona (Villegas, 2013). Una premisa que desde el surgimiento del enfoque humanista en psicología se ha venido promulgando, pero que, al momento de la practica terapéutica, suele estar obstaculizado por la prioridad de la técnica, que busca fortalecer la idea de la psicología como una ciencia basada en el método científico y no en lo humano. Siendo este uno de sus mayores focos de resistencia durante su surgimiento (Bugental, 1964).

\section{Conclusión}

Aunque la vivencia de la vulnerabilidad siempre implica malestar, desde la perspectiva existencial dicha vivencia va a depender del sentido y la forma de cómo cada persona la integra a la totalidad de su experiencia. Por un lado, podría permitir el continuo desarrollo personal, llevando a la persona a contemplar nuevas posibilidades de ser, ya sea resignificando valores o haciendo cambios de perspectiva, desde los más sencillos hasta los más trascendentales. Y, por otro lado, dado que no siempre se cuenta con los recursos psicológicos suficientes y necesarios para enfrentar la vida, la condición de vulnerabilidad tiende a frenarnos, lo que nos lleva a experimentar sensaciones de incapacidad personal, lo cual, muchas veces, podría llegar a paralizarnos, conduciéndonos a un progresivo distanciamiento de nuestras potencialidades, lo cual impide nuestro desarrollo. En estos momentos que se vivencian como críticos, tomaría relevancia el acompañamiento terapéutico, el encuentro empático, el establecimiento de una relación acogedora, y un terapeuta capaz de caminar al ritmo del paciente, aspectos fundamentales para el enfrentamiento de la situación a través de la resignificación de la experiencia y el desarrollo de los recursos psicológicos que sean necesarios para el consultante. 
Pues bien, hay quienes no pueden aflojar sus propias cadenas; sin embargo, pueden liberar a sus amigos (Zaratustra, citado por Yalom, 2008)

\section{Referencias}

Adger, W. N. (2006). Vulnerability. Global Environmental Change, 16, 268-281. https://doi.org/10.1016/j.gloenvcha.2006.02.006

Bugental, J. F. T. (1964). The Third Force in Psychology. Journal of Humanistic Psychology, 4(1), 19-26. https://doi. org/10.1177/002216786400400102

Cyrulnik, B. (2009). El murmullo de los fantasmas Volver a la vida después de un trauma. Madrid, España: Gedisa editorial.

de Castro, A. (2010). Importancia y sentido de la comprensión en psicología. Revista Latinoamericana De Psicología Existencial, 1(1), 14-19. Recuperado de http://www.fundacioncapac.org.ar/REVISTA_LATINOAMERICANA_1.pdf

de Castro Correa, A., \& García Chacón, G. (2011). Psicología Clínica Fundamentos existenciales (Segunda re). Barranquilla, Colombia: Editorial Uninorte.

Fromm, E. (2006). El Corazón del Hombre: Su Potencia Para el Bien y Para el mal. Barcelona, España: Fondo De Cultura Económica.

Horney, K. (1991). La Neurosis Y El Desarrollo Humano: La Lucha Por La Autorrealización. Buenos Aires, Argentina: Editorial Psique.

Martínez, Y. A. (2010). El Paradigma Relacional en la Psicoterapia Existencial. Revista Latinoamericana De Psicología Existencial, 1(1), 25-35. Recuperado de http://www.fundacioncapac.org.ar/REVISTA_LATINOAMERICANA_1.pdf

May, R. (1963). El hombre en busca de sí mismo. Madrid, España: Editorial Gredos.

May, R. (1974). Fuentes de la violencia. Buenos Aires, Argentina: Emecé Editores.

May, R. (1977). Existencia. Madrid, España: Editorial Gredos.

May, R. (1988). Libertad y destino en psicoterapia. Desclée de Brouwer. 
May, R. (1989). Psicología Existencial. Buenos Aires, Argentina: Editorial Paidós.

May, R. (1990). La psicología y el dilema del hombre. Barcelona, España: Gedisa.

May, R. (2000). Amor y voluntad: Las fuerzas que dan sentido a nuestra vida. México D.F:: Gedisa.

Riemann, F. (1996). Formas básicas de la angustia: estudio de psicología profunda. Barcelona, España: Herder.

Rispo, P. (2001). Por las ramas de la existencia: Fenomenología de las modalidades del ser. Buenos Aires, Argentina: Fundación CAPAC.

Rogers, C. R. (1981). Psicoterapia Centrada en el Cliente. Barcelona, España: Paidós.

Rogers, C. R. (2014). Terapia, Personalidad y Relaciones Interpersonales (8a ed.). Buenos Aires: Nueva Visión.

Romero, E. (2003). El inquilino de lo imaginario, formas malogradas de existencia. (la ed.). Ediciones Norte - Sur. Peñaflor, Chile. Romero, E. (2003). Neogénesis, el desarrollo personal mediante la psicoterapia. Sao Pablo: Editorial Norte - Sur (b).

Villegas Besora, M. (2013). Prometeo en el diván Psicoterapia del desarrollo moral. Barcelona, España: Herder Editorial.

Yalom, I. D. (1997). Desde el diván. Buenos Aires, Argentina: Emecé Editores.

Yalom, I. D. (2002). El don de la terapia: carta abierta a una nueva generación de terapeutas y a sus pacientes. Buenos Aires, Argentina: Emecé Editores.

Yalom, I. D. (2008). El día que Nietzsche lloró. Barcelona, España: Destino.

Yalom, I. D. (2009). Mirar al sol : la superación del miedo a la muerte. Buenos Aires, Argentina: Booket.

Yalom, I. D. (2011). Psicoterapia existencial (2da ed.). Barcelona, España: Herder Editorial.

Yalom, I. D., \& Elkin, G. (2007). Terapia a dos voces. Buenos Aires, Argentina: Emecé Editores. 